\title{
A comparison of symmetry and flatness measurements in small electron fields by different dosimeters in electron beam radiotherapy
}

\author{
Mohamad Reza Bayatiani ${ }^{1}$, Fatemeh Fallahi', Akbar Aliasgharzadeh ${ }^{2}$, Mahdi Ghorbani', \\ Benyamin Khajetash $^{4}$, Fatemeh Seif ${ }^{1}$ \\ ${ }^{1}$ Medical Physics and Radiotherapy Department, School of Paramedical Sciences, \\ Arak University of Medical Sciences and Khansari Hospital, Arak, Iran \\ ${ }^{2}$ Department of Medical Physics, School of Paramedical Sciences, Kashan University of Medical Sciences, Kashan, Iran \\ ${ }^{3}$ Biomedical Engineering and Medical Physics Department, School of Medicine, Shahid Beheshti University of Medical Sciences, Tehran, Iran \\ ${ }^{4}$ Medical Physics Department, School of Medicine, Iran University of Medical Sciences, Tehran, Iran
}

\begin{abstract}
Background: Symmetry and flatness are two quantities which should be evaluated in the commissioning and quality control of an electron beam in electron beam radiotherapy. The aim of this study is to compare symmetry and flatness obtained using three different dosimeters for various small and large fields in electron beam radiotherapy with linac.

Materials and methods: Beam profile measurements were performed in a PTW water phantom for 10, 15 and $18 \mathrm{MeV}$ electron beams of an Elekta Precise linac for small and large beams $\left(1.5 \times 1.5 \mathrm{~cm}^{2}\right.$ to $20 \times 20 \mathrm{~cm}^{2}$ field sizes). A Diode E detector and Semiflex-3D and Advanced Markus ionization chambers were used for dosimetry.

Results: Based on the obtained results, there are minor differences between the responses from different dosimeters (Diode E detector and Semiflex-3D and Advanced Markus ionization chambers) in measurement of symmetry and flatness for the electron beams. The symmetry and flatness values increase with increasing field size and electron beam energy for small and large field sizes, while the increases are minor in some cases.

Conclusions: The results indicate that the differences between the symmetry and flatness values obtained from the three dosimeter types are not practically important.
\end{abstract}

Key words: radiotherapy; small field dosimetry; electron beam; symmetry; flatness; Diode E; Semiflex-3D; Advanced Markus Rep Pract Oncol Radiother 2021;26(1):50-58

\section{Introduction}

Electron beam in radiotherapy, due to its characteristics, is appropriate for treatment of superficial tumors and sparing deep tissues. One of the most prominent features of electron beam is relatively uniform dose delivery from body surface to a specific depth (which depends on electron energy) and rapid dose fall-off in depth [1].
Quality control in radiotherapy has an important role and obtaining data for electron beams through quality control with proper and accurate tools is vital to provide adequate treatment outcomes. Symmetry and flatness are two variables which are normally measured as part of quality control. Selection of adequate and accurate radiation detector among a broad range of accessible systems covering all sizes (regular, mini-, to micro- 
detectors) and types (ionization chambers, semiconductors, etc.) is crucial to obtain a beam profile that is the closest possible data to the real one [2]. Radiation detectors have a vigorous measuring volume of finite size causing them to own a finite spatial resolution. This limitation is more pronounced in high dose gradient regions and might lead to a synthetic increase of the penumbra width. This effect, which is known in the literature as "the volume averaging effect", plays an important role in the choice of a suitable detector and has been the topic of many publications $[3,4]$.

In radiotherapy, a major requirement is that the dose variation over the target volume is restricted so that all points within the volume receive the prescribed dose within a tolerance range. Symmetry and flatness are two quantities which have effect on the uniformity of dose in target volume. American Association of Physicists in Medicine (AAPM) in task group No. 25 report has recommendations regarding clinical dosimetry for electron beams [5]. This task group published a supplement to this report in 2009 [6]. There are also issues on small field dosimetry for electron beams, which is a challenging subject, in these reports. There are various studies in which symmetry and flatness of different linacs were determined as part of the study [7-15]. In these studies, different linac models and dosimetry methods were evaluated.

Generally, there are several challenges in selection of a detector in radiation dosimetry, especially in electron small fields. In clinical dosimetry data entry in treatment planning systems this is a very important issue because dose distribution and patient calculations depend strongly on these data. Therefore, choosing an adequate dosimeter for such measurements is very important. For example, in beam profiles, at the penumbra region, measuring the dose is crucial because of high dose gradient. This will be more important in small fields in electron beams. All dosimetry equipment companies update their products and produce new dosimeters. One of these interesting dosimeters is Semiflex-3D (release 2016) that is used for measurements and is compared with routine dosimeters, such as Advanced Markus and Diode E detectors in the present study. The aim of the present study is to compare symmetry and flatness values in small electron beams by different dosimeters in electron beam radiotherapy with an Elekta Precise linac.

\section{Materials and methods}

In current study, symmetry and flatness was measured for an Elekta Precise linac based on the International Electrotechnical Commission (IEC) protocol [16] for electron dosimetry. The irradiations were performed by 10,15 and $18 \mathrm{MeV}$ electron beams of an Elekta Precise linear accelerator. The measurements were performed in a MP3-M water phantom (PTW, Freiburg, Germany) in Ayatollah Khansari Hospital (Arak, Iran). Diode E (PTW, Freiburg, Germany), Semiflex-3D ionization chamber (PTW, Freiburg, Germany) and Advanced Markus parallel plate ionization chamber (PTW, Freiburg, Germany) were used for measurements. The sensitive volume of the Diode E detector is $0.03 \mathrm{~mm}^{3}$. The sensitive volume for the Semiflex-3D and Advanced Markus chambers are $0.07 \mathrm{~cm}^{3}, 0.02$ $\mathrm{cm}^{3}$, respectively. A UNIDOS E electrometer (PTW, Freiburg, Germany) was utilized for reading the responses of these detectors. These detectors have been calibrated by a secondary standards dosimetry laboratory (SSDL). This laboratory is traceable to the Atomic Energy Organization of Iran (AEOI). Symmetry and flatness for different small and large fields were evaluated. The small fields included 1.5 $\times 1.5,2 \times 2,2.5 \times 2.5,3 \times 3,3.5 \times 3.5,4 \times 4,4.5 \times$ $4.5,5 \times 5 \mathrm{~cm}^{2}$ fields, and the large fields included 6 $\times 6,10 \times 10,14 \times 14$ and $20 \times 20 \mathrm{~cm}^{2}$ fields.

Based on the IEC protocol, symmetry of a radiation field is defined as the maximum dose ratio at two symmetric points relative to the field's central axis [16]:

$$
\operatorname{Symmetry}(\%)=\left|\frac{D_{(x . y)}}{D_{(-x-y)}}\right|_{\max } \times 100
$$

According to this protocol, flatness of a beam is defined by the Formula number (2):

$$
\text { Flatness }(\%)=\frac{D_{\max }}{D_{\min }} \times 100
$$

where $D_{\max }$ and $D_{\min }$ are the maximum and minimum doses with the beam profile at a depth of interest, respectively. To obtain symmetry and flatness at the depth of maximum dose $\left(d_{\max }\right)$, beam profile at $d_{\max }$ was measured for the mentioned electron beam energies and field sizes with the three dosimeters. The $d_{\max }$ for the energy of 10,15 and 


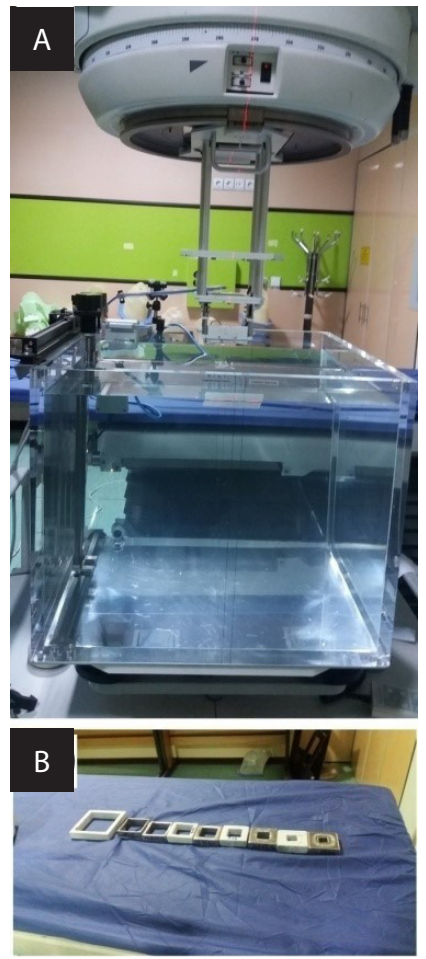

Figure 1. A typical set-up for the measurements (A) and the cutouts (B)

$18 \mathrm{MeV}$ were $2.2,2.51$ and $2.72 \mathrm{~cm}$, respectively. The irradiation for measurement for each field was performed by enough monitor units (MU) so that it can cover the whole field size and an adequate field margin. The measurements were performed at the source to surface distance (SSD) of $100 \mathrm{~cm}$. To increase the repeatability, measurements were repeated three times. The measurements were performed in $1 \mathrm{~mm}$ steps inside the phantom up to $3 \mathrm{~cm}$ distance outside each field edge, in which the dosimeter reading was zero. The measurements were performed in in-line direction. In Figure 1, a typical set-up for the measurements and cutouts are presented. Small fields were shaped using $6 \times 6$ $\mathrm{cm}^{2}$ applicator and cerrobend cutouts. The cutouts had $1 \mathrm{~cm}$ thickness.

\section{Results}

Sample beam profiles for $2 \times 2,3 \times 3,6 \times 6$ and $10 \times 10 \mathrm{~cm}^{2}$ fields are presented in Figure 2. Results of symmetry and flatness obtained from Diode E dosimeter, Semiflex-3D and Advanced Markus ionization chambers for small and large fields in 10, 15, and $18 \mathrm{MeV}$ electron beam energies are presented in Tables 1, 2 and 3.

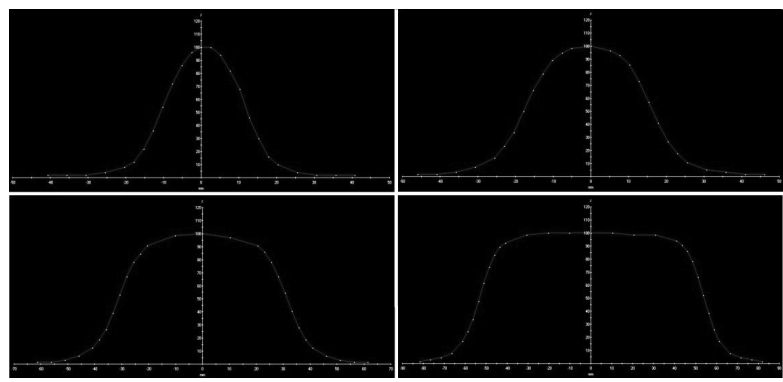

Figure 2. Sample beam profiles for $2 \times 2,3 \times 3,6 \times 6$ and $10 \times 10 \mathrm{~cm}^{2}$ fields, obtained from in-phantom measurements

Results of symmetry (\%) obtained from Diode E detector, and Semiflex-3D and Advanced Markus ionization chambers in small fields (a) and large fields (b) in $18 \mathrm{MeV}$ electron beam energy are presented in Figure 3. Results of flatness (\%) obtained from Diode E detector, and Semiflex-3D and Advanced Markus ionization chambers in small fields (a) and large fields (b) in $18 \mathrm{MeV}$ electron beam energy are presented in Figure 4. Percentage differences (\%) between symmetry and flatness values obtained from Diode E detector, and Semiflex-3D and Advanced Markus ionization chambers in small fields and large fields in $18 \mathrm{MeV}$ electron beam energy are presented in Table 4 .

\section{Discussion}

In the present study, symmetry and flatness were obtained using three different dosimeters for various small and large fields in electron beam radiotherapy in 10,15 and $18 \mathrm{MeV}$ electron beams of an Elekta Precise linac. Based on the results presented in Tables 1, 2 and 3, in most of the data points, the values of symmetry and flatness are within the acceptable criteria (103\% for both symmetry and flatness) according to the IEC protocol [16]. The data points which exceed these criteria are mostly related to flatness, large fields and higher electron energies. These results also indicate that there are minor differences between the symmetry and flatness values obtained from different dosimeters (Diode E detector and Semiflex-3D and Advanced Markus ionization chambers). The results of symmetry and flatness obtained from Diode E detector, and Semiflex-3D and Advanced Markus ionization chambers in small fields (a) and large fields (b) in $18 \mathrm{MeV}$ electron beam energy are presented in Fig. 3 and Fig. 4, respectively. This energy was selected 
Table 1. Results of symmetry and flatness obtained from Diode E dosimeter in small and large fields in 10, 15, and $18 \mathrm{MeV}$ electron beam energies

\begin{tabular}{|c|c|c|c|c|c|c|c|}
\hline & \multirow{2}{*}{ Field size $\left[\mathrm{cm}^{2}\right]$} & \multicolumn{2}{|c|}{$10 \mathrm{MeV}$} & \multicolumn{2}{|c|}{$15 \mathrm{MeV}$} & \multicolumn{2}{|c|}{$18 \mathrm{MeV}$} \\
\hline & & Symmetry (\%) & Flatness (\%) & Symmetry (\%) & Flatness (\%) & Symmetry (\%) & Flatness (\%) \\
\hline \multirow{8}{*}{$\begin{array}{l}\text { Small } \\
\text { fields }\end{array}$} & $1.5 \times 1.5$ & 100.19 & 100.28 & 100.22 & 100.54 & 100.28 & 100.62 \\
\hline & $2 \times 2$ & 100.23 & 100.34 & 100.24 & 100.69 & 100.36 & 100.94 \\
\hline & $2.5 \times 2.5$ & 100.33 & 100.86 & 100.35 & 101.75 & 100.48 & 102.05 \\
\hline & $3 \times 3$ & 100.35 & 101.91 & 100.51 & 102.05 & 100.56 & 102.14 \\
\hline & $3.5 \times 3.5$ & 100.43 & 102.35 & 100.63 & 102.53 & 100.67 & 102.69 \\
\hline & $4 \times 4$ & 100.72 & 102.98 & 100.77 & 103.21 & 100.82 & 103.52 \\
\hline & $4.5 \times 4.5$ & 100.79 & 103.33 & 100.96 & 103.64 & 101.39 & 104.10 \\
\hline & $5 \times 5$ & 100.86 & 103.54 & 101.01 & 103.83 & 101.56 & 104.82 \\
\hline \multirow{4}{*}{$\begin{array}{l}\text { Large } \\
\text { fields }\end{array}$} & $6 \times 6$ & 101.01 & 104.61 & 101.12 & 104.72 & 101.79 & 105.23 \\
\hline & $10 \times 10$ & 101.73 & 105.14 & 101.84 & 105.33 & 102.24 & 106.12 \\
\hline & $14 \times 14$ & 101.87 & 106.43 & 101.91 & 107.47 & 102.42 & 107.63 \\
\hline & $20 \times 20$ & 102.53 & 108.24 & 102.48 & 108.37 & 103.02 & 108.87 \\
\hline \multicolumn{2}{|c|}{ Range (\%) } & $100.19-102.53$ & $100.28-18.24$ & $100.22-102.48$ & $100.54-108.37$ & $100.28-103.02$ & $100.62-108.87$ \\
\hline
\end{tabular}

Table 2. Results of symmetry and flatness obtained from Semiflex-3D ionization chamber in small and large fields in 10, 15, and $18 \mathrm{MeV}$ electron beam energies

\begin{tabular}{|c|c|c|c|c|c|c|c|}
\hline & \multirow{2}{*}{ Field size $\left[\mathrm{cm}^{2}\right]$} & \multicolumn{2}{|c|}{$10 \mathrm{MeV}$} & \multicolumn{2}{|c|}{$15 \mathrm{MeV}$} & \multicolumn{2}{|c|}{$18 \mathrm{MeV}$} \\
\hline & & Symmetry (\%) & Flatness (\%) & Symmetry (\%) & Flatness (\%) & Symmetry (\%) & Flatness (\%) \\
\hline \multirow{8}{*}{$\begin{array}{l}\text { Small } \\
\text { fields }\end{array}$} & $1.5 \times 1.5$ & 100.05 & 100.15 & 100.19 & 100.32 & 100.12 & 100.37 \\
\hline & $2 \times 2$ & 100.08 & 100.27 & 100.22 & 100.83 & 100.13 & 100.97 \\
\hline & $2.5 \times 2.5$ & 100.13 & 101.15 & 100.30 & 101.51 & 100.37 & 101.83 \\
\hline & $3 \times 3$ & 100.20 & 101.60 & 100.39 & 101.75 & 100.47 & 102.01 \\
\hline & $3.5 \times 3.5$ & 100.43 & 102.33 & 100.50 & 102.41 & 100.67 & 103.86 \\
\hline & $4 \times 4$ & 100.52 & 103.03 & 100.63 & 103.68 & 100.88 & 104.21 \\
\hline & $4.5 \times 4.5$ & 100.75 & 104.01 & 100.79 & 104.43 & 101.02 & 104.64 \\
\hline & $5 \times 5$ & 100.80 & 104.32 & 100.85 & 104.92 & 101.26 & 105.87 \\
\hline \multirow{4}{*}{$\begin{array}{l}\text { Large } \\
\text { fields }\end{array}$} & $6 \times 6$ & 100.91 & 104.79 & 101.08 & 105.40 & 101.37 & 106.46 \\
\hline & $10 \times 10$ & 101.56 & 105.07 & 101.64 & 105.88 & 101.89 & 106.99 \\
\hline & $14 \times 14$ & 101.89 & 106.98 & 101.98 & 107.11 & 102.10 & 107.54 \\
\hline & $20 \times 20$ & 102.38 & 108.05 & 102.45 & 108.32 & 102.55 & 109.28 \\
\hline \multicolumn{2}{|c|}{ Range (\%) } & $100.05-102.38$ & $100.15-108.05$ & $100.19-102.45$ & $100.32-108.32$ & $100.12-102.55$ & $100.37-109.28$ \\
\hline
\end{tabular}

for presentation of these figures and comparison of the responses of the detectors because the difference between the responses was more dominant in this energy. The minor difference (Tab. 4) in the results of symmetry and flatness obtained from different detectors may be due to the sensitive volumes and materials of the detectors. The sensitive volume of the Diode E detector is $0.03 \mathrm{~mm}^{3}$. The sensitive volumes for the Semiflex-3D and Advanced Markus chambers are $0.07 \mathrm{~cm}^{3}, 0.02 \mathrm{~cm}^{3}$, respectively. Basi- cally, the smaller sensitive volume of Diode E detector corresponds to a lower volume averaging effect. In small fields, this can be assumed as an advantage for diode detectors versus ionization chambers [2]. Generally, semiconductor detectors have a higher atomic number and this may have effects on their response. It should also be noted that the sensitive volume is important in measurement of absorbed dose in the penumbra region, which has not a major relevance in the present study because symme- 
Table 3. Results of symmetry and flatness obtained from Advanced Markus ionization chamber in small and large fields in 10, 15 , and $18 \mathrm{MeV}$ electron beam energies

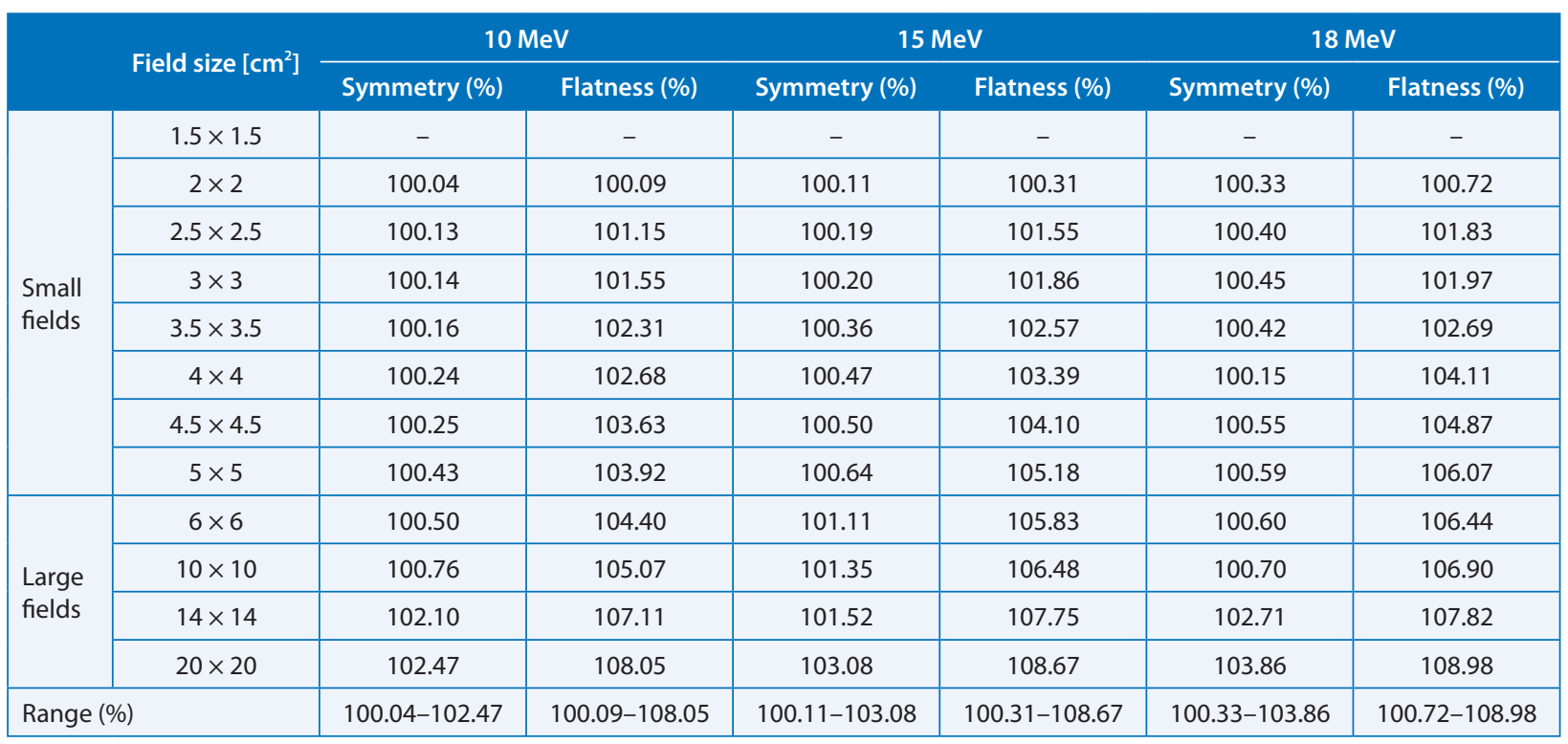

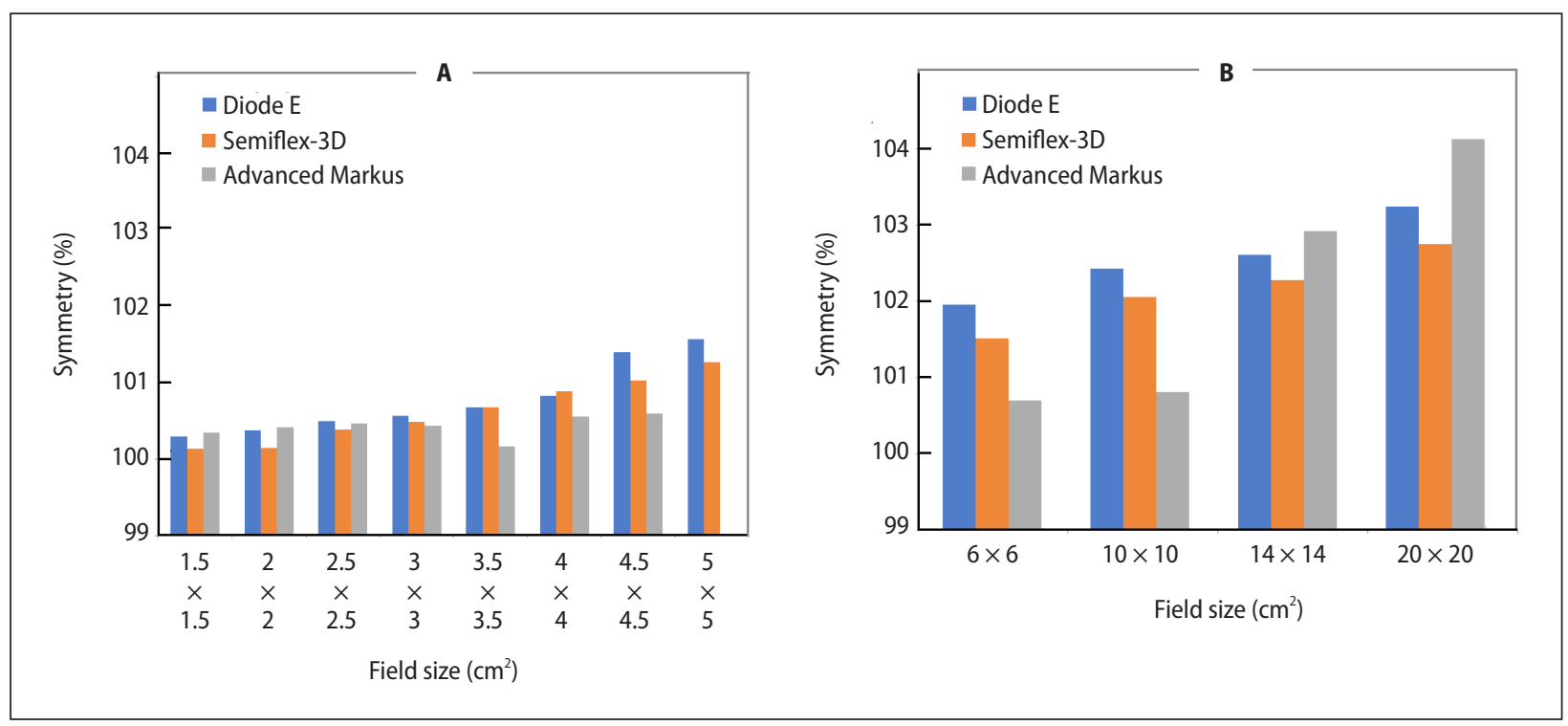

Figure 3. Results of symmetry (\%) obtained from Diode E detector, and Semiflex-3D and Advanced Markus ionization chambers in small fields (A) and large fields $(\mathbf{B})$ in $18 \mathrm{MeV}$ electron beam energy

try and flatness are measured by dosimetry of the beam profile in the flat part of the beam profile.

For small fields, the symmetry values have minor increases with increasing field size for all three detectors in 10, 15 and $18 \mathrm{MeV}$ beam energies. For a given field size, the symmetry also has minor increases with increasing electron beam energy. This trend is observed for all the three detectors. For small fields, the flatness values increase with increasing field size for all three detectors in 10, 15 and $18 \mathrm{MeV}$ beam energies. For a given field size, the flatness also has minor increases with increasing electron beam energy. This trend is observed for all the three detectors. Small field dosimetry is a challenging subject in electron beam dosimetry. When the size of a field is smaller than the radius needed for lateral equilibrium, there is a change in $d_{\max }$, percent depth dose, and output. When the field size is smaller, the dose distribution changes in all directions. Therefore, for small fields it is recom- 


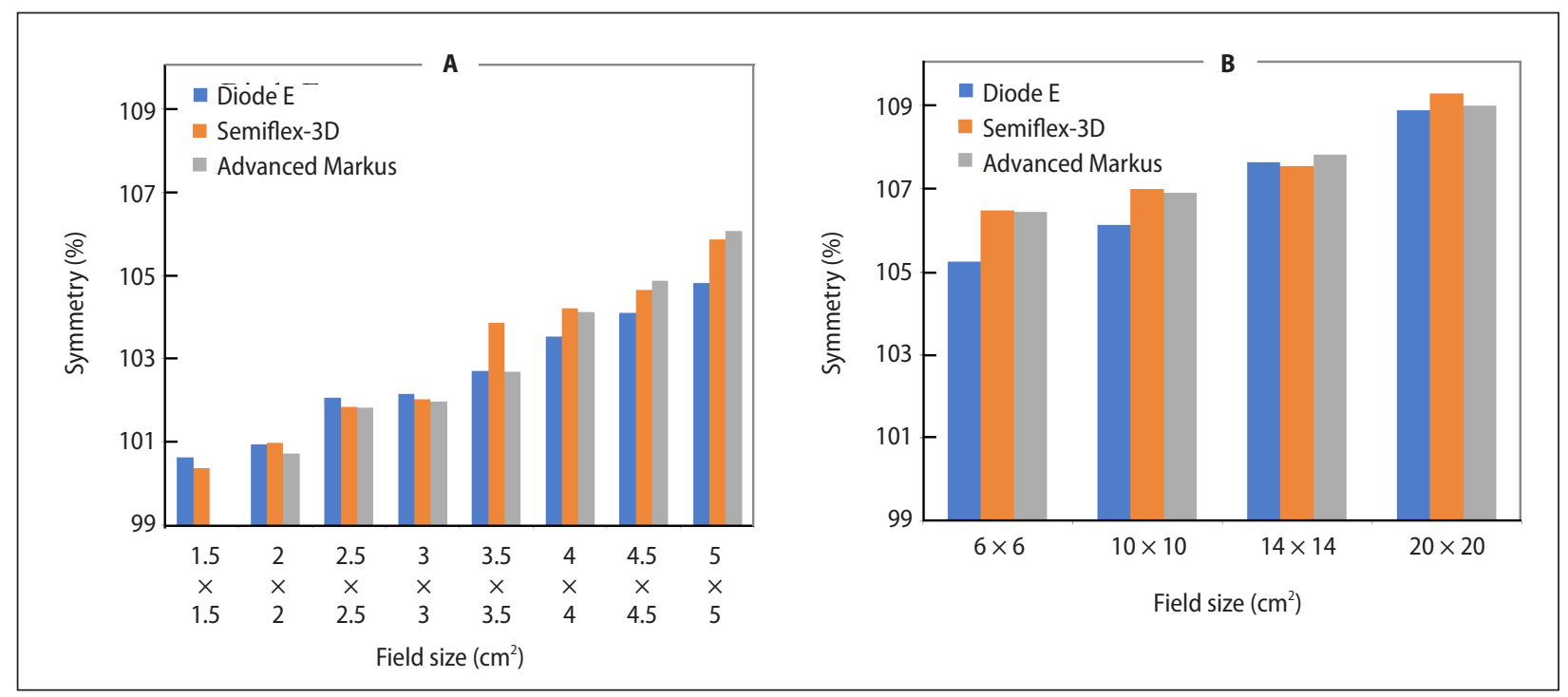

Figure 4. Results of flatness (\%) obtained from Diode E detector, and Semiflex-3D and Advanced Markus ionization chambers in small fields (A) and large fields (B) in $18 \mathrm{MeV}$ electron beam energy

Table 4. Percentage difference (\%) between symmetry and flatness values obtained from Diode E detector, and Semiflex and Advanced Markus ionization chambers in small fields and large fields in $18 \mathrm{MeV}$ electron beam energy

\begin{tabular}{|c|c|c|c|c|c|}
\hline & \multirow[b]{2}{*}{ Field size $\left[\mathrm{cm}^{2}\right]$} & \multicolumn{2}{|c|}{ Symmetry } & \multicolumn{2}{|c|}{ Flatness } \\
\hline & & Diode E-Semiflex & $\begin{array}{c}\text { Diode E-Advanced } \\
\text { Markus }\end{array}$ & Diode E-Semiflex & $\begin{array}{c}\text { Diode E-Advanced } \\
\text { Markus }\end{array}$ \\
\hline \multirow{8}{*}{$\begin{array}{l}\text { Small } \\
\text { fields }\end{array}$} & $1.5 \times 1.5$ & 0.16 & - & 0.25 & - \\
\hline & $2 \times 2$ & 0.23 & 0.03 & -0.03 & 0.22 \\
\hline & $2.5 \times 2.5$ & 0.11 & 0.08 & 0.22 & 0.22 \\
\hline & $3 \times 3$ & 0.09 & 0.11 & 0.13 & 0.17 \\
\hline & $3.5 \times 3.5$ & 0.00 & 0.25 & -1.14 & 0.00 \\
\hline & $4 \times 4$ & -0.06 & 0.66 & -0.67 & -0.57 \\
\hline & $4.5 \times 4.5$ & 0.36 & 0.83 & -0.52 & -0.74 \\
\hline & $5 \times 5$ & 0.30 & 0.96 & -1.00 & -1.19 \\
\hline \multirow{4}{*}{$\begin{array}{l}\text { Large } \\
\text { fields }\end{array}$} & $6 \times 6$ & 0.41 & 1.17 & -1.17 & -1.15 \\
\hline & $10 \times 10$ & 0.34 & 1.51 & -0.82 & -0.74 \\
\hline & $14 \times 14$ & 0.31 & -0.28 & 0.08 & -0.18 \\
\hline & $20 \times 20$ & 0.46 & -0.82 & -0.38 & -0.10 \\
\hline
\end{tabular}

mended that custom measurements be performed for central axis dose, output and dose profiles. For this purpose, an ionization chamber, diode and film dosimeters are proposed. There are also mathematical approaches for determination of electron dose distribution in the literature [6]. The data in the present study are custom measurements for Elekta Precise linac, and since commissioning should be performed for each linac in each radiotherapy center independently, the data cannot be used for other linac models or other Elekta Precise linacs. However, the data presented herein are illuminating as the symmetry and flatness data for small and large fields for an Elekta Precise linac.

For large fields, the symmetry values increase with increasing field size for all three detectors in 10,15 and $18 \mathrm{MeV}$ beam energies. For a given field size, the symmetry has minor increases with increasing electron beam energy. This trend is observed for all the three detectors. For large fields, 
the flatness values increase with increasing field size for all three detectors in 10,15 and $18 \mathrm{MeV}$ beam energies. For a given field size, the flatness also has minor increases with increasing electron beam energy. This trend is observed for all the three detectors.

The main cause of problems in symmetry and flatness of electron beams is due to misalignment of the central axis of the beam with the center of scattering foil. Nonuniformity in the thickness of scattering foil can also cause problems in symmetry and flatness. However, these problems are not relevant to the results of symmetry and flatness in this study, because major problems are solved in the step of acceptance test of a radiotherapy linac. Additionally, the symmetry and flatness values in the present study are mostly acceptable.

In the dosimetry process one can use different dosimeters for measurement of absorbed dose, but it is noticeable that radiation detection for dosimetric purposes in the field of diagnostic radiology, radiotherapy and nuclear medicine is mainly based on three principles of measurement, realized by three different detector types: ionization chamber, silicon diode detector and synthetic diamond diode detector. In fact, the responses of these detectors in electron beam dosimetry depend on the physics of electron interactions, such as collision and bremsstrahlung. In other words, electron has two types of interactions with material: collision and bremsstrahlung. The most probable electron interaction is collision, and bremsstrahlung interaction has low probability, which shows its effect on the tail part of the percent depth dose curve, and it has minor contribution in the dose. The most electron collisions occur due to interaction with scattering foil. Collision with phantom material also occurs but it has no effect on symmetry and flatness results in the present study because the phantom material was water and it was homogeneous and uniform. Since most symmetry and flatness results are acceptable based on standard criteria, these issues cannot be sources of problem in the present results of symmetry and flatness measured with different dosimeters.

The Advanced Markus (a routine dosimeter for electron field dosimetry) and Semiflex-3D (a new product of PTW) that were used in this study are ionization chambers. An ionization chamber basically consists of a gas volume between two electrodes connected to a high voltage. After exposing to radiation, ion pairs are created in the gas volume and the positive and negative charges are attracted by the electrodes, thus, creating a charge or current which can be measured by an electrometer. The diode used in this study (Diode E) is a silicon semiconductor that has a layer of n-type silicon which is brought into contact with a layer of p-type silicon, allowing electrons to drift from the $\mathrm{n}$ to the $\mathrm{p}$ region. Incident radiation frees electrons in the intrinsic zone (the sensitive layer of the detector) which move toward the positively charged $\mathrm{p}$ region, generating a current. This solar cell principle does not need an external bias voltage. Since selection of an adequate detector for dosimetry, especially in electron fields, is challenging, the present study aimed to compare the results of dosimetry of electron profiles with different dosimeters.

There are various studies in which symmetry and flatness of different linacs were determined as part of the study [7-15]. Patatoukas et al. [2] have determined profile characteristics for electron beams using different dosimeters including a Semiflex$-3 \mathrm{D}$, a Markus and a Roos ionization chambers, a p-type and an e-type diodes and one dimensional Linear Array. Their findings have shown that for symmetry the Linear Array shows the most symmetric beam profiles. This is due to the simultaneous measurements of different parts of beam profile by Linear Array while stepping measurement by other dosimeters may be accompanied by variations in the linac output during the time period of measurement of different parts of the beam profile. Regarding flatness, the detectors which showed larger penumbra resulted in higher flatness values. A limitation of that study is that it does not clearly indicate which detector has the closest results to the true values regarding the penumbra region, symmetry and flatness [2]. This is also true for the results of the present study, for which the results are practically equal for different dosimeters and it is impossible to determine the most accurate dosimeter. To the best of our knowledge, there is no similar study on symmetry and flatness determined by these dosimeters for the results of the current study to be compared with. Performing corresponding dosimetry with other techniques, such as radiochromic film dosimetry, Monte Carlo simulation, 
and analytical approach is suggested as future studies in this field.

In a study by Kądziołka et al. [17] dosimetric characteristics of photon and electron beams of an Elekta Precise linac was evaluated. A Farmer-type ionization chamber was used for the measurements. Those electron energies and field sizes which are similar to the present study was $15,18 \mathrm{MeV}$ and 10 $\times 10 \mathrm{~cm}^{2}$. The symmetry and flatness values in 15 $\mathrm{MeV}$ energy was $100.49 \%$ and $103.26 \%$, respectively, and in $18 \mathrm{MeV}$ energy it was $100.70 \%$ and $102.72 \%$, respectively. There are differences between these values and the corresponding values in the present study. Additionally, in that study the symmetry and flatness decreased with electron energy and there were minor changing trends with increasing field size from $10 \times 10 \mathrm{~cm}^{2}$ to $25 \times 25 \mathrm{~cm}^{2}$, while the trends in the present study are in some extent different. In that study, only $10 \times 10 \mathrm{~cm}^{2}$ and $25 \times 25 \mathrm{~cm}^{2}$ fields were evaluated for electron beams.

\section{Conclusion}

Based on the obtained results, there are minor differences between the responses from different dosimeters (Diode E detector and Semiflex-3D and Advanced Markus ionization chambers) in measurement of symmetry and flatness for the electron beams of 10,15 , and $18 \mathrm{MeV}$ energies. The results indicate these differences are not practically important. The symmetry and flatness values increase with increasing field size and electron beam energy for small and large field sizes, while the increases are minor in some cases.

\section{Conflict of interest}

There is not any relationship that might lead to a conflict of interest.

\section{Funding}

Arak University of Medical Sciences has financially supported the work.

\section{Acknowledgement}

The authors would like to thank Department of Radiotherapy and Oncology of Ayatollah Khansari Hospital (Arak, Iran) for technical support of this work. This study was supported by Arak University of Medical Sciences with a grant number of 1232 and an ethical code of IR.ARAKMU.REC.1395.223.

\section{References}

1. Strydom W, Parker W, Olivares M. Electron beams: physical and clinical aspects. In: Podgorsak EB. ed. Radiation oncology physics: a handbook for teachers and students. International Atomic Energy Agency (IAEA), Vienna 2005: 273.

2. Patatoukas GD, Kalavrezos $P$, Seimenis $I$, et al. Determination of beam profile characteristics in radiation therapy using different dosimetric set ups. J BUON. 2018; 23: 1448-1459, indexed in Pubmed: 30570872.

3. Benítez EM, Casado FJ, García-Pareja S, et al. Evaluation of a liquid ionization chamber for relative dosimetry in small and large fields of radiotherapy photon beams. Radiat Meas. 2013; 58: 79-86, doi: 10.1016/j.radmeas.2013.08.009.

4. García-Vicente F, Béjar MJ, Pérez L, et al. Clinical impact of the detector size effect in 3D-CRT. Radiother Oncol. 2005; 74(3): 315-322, doi: 10.1016/j.radonc.2004.10.012, indexed in Pubmed: 15763313.

5. Khan FM, Doppke KP, Hogstrom KR, et al. Clinical electronbeam dosimetry: report of AAPM Radiation Therapy Committee Task Group No. 25. Med Phys. 1991; 18(1): 73-109, doi: 10.1118/1.596695, indexed in Pubmed: 1901132.

6. Gerbi BJ, Antolak JA, Deibel FC, et al. Recommendations for clinical electron beam dosimetry: supplement to the recommendations of Task Group 25. Med Phys. 2009; 36(7): 3239-3279, doi: 10.1118/1.3125820, indexed in Pubmed: 19673223.

7. Baghani HR, Aghamiri SM, Mahdavi SR, et al. Comparing the dosimetric characteristics of the electron beam from dedicated intraoperative and conventional radiotherapy accelerators. J Appl Clin Med Phys. 2015; 16(2): 5017, doi: 10.1120/jacmp.v16i2.5017, indexed in Pubmed: 26103175.

8. Binny D, Lancaster CM, Kairn T, et al. Monitoring Daily QA 3 constancy for routine quality assurance on linear accelerators. Phys Med. 2016; 32(11): 1479-1487, doi: 10.1016/j. ejmp.2016.10.021, indexed in Pubmed: 27839928.

9. Chan MF, Li Q, Tang X, et al. Visual Analysis of the Daily QA Results of Photon and Electron Beams of a Trilogy Linac over a Five-year Period. Int J Med Phys Clin Eng Radiat Oncol. 2015; 4(4): 290-299, doi: 10.4236/ijmpcero.2015.44035, indexed in Pubmed: 27547595.

10. Klein EE, Low DA, Purdy JA. Changes in electron beam dosimetry with a new scattering foil-applicator system on a CL2100C. Int J Radiat Oncol Biol Phys. 1995; 32(2): 483-490, doi: 10.1016/0360-3016(94)00452-Q, indexed in Pubmed: 7751189.

11. Narayanasamy G, Saenz D, Cruz W, et al. Commissioning an Elekta Versa HD linear accelerator. J Appl Clin Med Phys. 2016; 17(1): 179-191, doi: 10.1120/jacmp.v17i1.5799, indexed in Pubmed: 26894351.

12. Nicewonger D, Stathakis PM. PTW QUICKCHECKwebline: Daily quality assurance phantom comparison and overall performance. J BUON. 2019; 24(4): 1727-1734, indexed in Pubmed: 31646832.

13. Park JM, Park SY, Chun M, et al. On-site audits to investigate the quality of radiation physics of radiation therapy institutions in the Republic of Korea. Phys Med. 2017; 40: 110-114, doi: 10.1016/j.ejmp.2017.07.021, indexed in Pubmed: 28784573. 
14. Watts RJ. Evaluation of a diode detector array for use as a linear accelerator QC device. Med Phys. 1998; 25(2): 247250, doi: 10.1118/1.598188, indexed in Pubmed: 9507488.

15. Yaddanapudi S, Cai B, Harry T, et al. Rapid acceptance testing of modern linac using on-board $\mathrm{MV}$ and $\mathrm{kV}$ imaging systems. Med Phys. 2017; 44(7): 3393-3406, doi: 10.1002/ mp.12294, indexed in Pubmed: 28432806.
16. IEC. Medical electron accelerators - functional performance characteristics. International Electrotechnical Commission, Geneva 1989.

17. Kądziołka E, Kisielewska-Birycka M, Surowiak T, et al. Information about the first Elekta Precise ${ }^{\circledR}$ accelerator installed in Poland. Rep Pract Oncol Radiother. 2006; 11(3): 147-155, doi: 10.1016/s1507-1367(06)71060-4. 